Bull. Korean Math. Soc. 48 (2011), No. 5, pp. 951-957

http://dx.doi.org/10.4134/BKMS.2011.48.5.951

\title{
A NOTE ON THE BRÜCK CONJECTURE
}

\author{
FENG Lü
}

\begin{abstract}
In 1996, Brück studied the relation between $f$ and $f^{\prime}$ if an entire function $f$ shares one value $a \mathrm{CM}$ with its first derivative $f^{\prime}$ and posed the famous Brück conjecture. In this work, we generalize the value $a$ in the Brück conjecture to a small function $\alpha$. Meanwhile, we prove that the Brück conjecture holds for a class of meromorphic functions.
\end{abstract}

\section{Introduction and main results}

To state our main result, we need the following concepts and definitions.

Definition 1. The order $\rho(f)$ and the super order $\sigma_{2}(f)$ of a meromorphic function $f$ are defined by

$$
\rho(f)=\varlimsup_{r \rightarrow \infty} \frac{\log T(r, f)}{\log r}, \sigma_{2}(f)=\varlimsup_{r \rightarrow \infty} \frac{\log \log T(r, f)}{\log r}
$$

respectively.

Let $f, g$ and $\alpha$ be three meromorphic functions in the complex plane $\mathbb{C}$. We say $f$ and $g$ share $\alpha$ CM provided that $f-\alpha$ and $g-\alpha$ have the same zeros with the same multiplicities. If $f-\alpha$ and $g-\alpha$ have the same zeros, then we say $f$ and $g$ share $\alpha$ IM and denote it by $f(z)=\alpha(z) \Leftrightarrow g(z)=\alpha(z)$. It is assumed that the reader is familiar with the standard symbols and fundamental results of Nevanlinna theory, as found in $[6,13]$.

The subject on sharing values between entire functions and their derivatives was first studied by Rubel and Yang [11]. They proved a result in 1977 that if a non-constant entire function $f$ and its first derivative $f^{\prime}$ share two distinct finite numbers $a, b \mathrm{CM}$, then $f=f^{\prime}$. Since then, shared value problems have been studied by many authors and a number of profound results have been obtained(see, e.g., $[5,10])$.

Received January 21, 2010; Revised March 19, 2010.

2010 Mathematics Subject Classification. 30D35, 30D45.

Key words and phrases. entire functions, Nevanlinna theory, uniqueness, normal family.

The research was supported by the NSFC Tianyuan Mathematics Youth Fund (No.11026146) and the Fundamental Research Funds for the Central Universities (No.10CX04038A). 
In 1996, Brück [1] studied the relation between $f$ and $f^{\prime}$ if an entire function $f$ shares one value $\mathrm{CM}$ with its first derivative $f^{\prime}$. Meanwhile, he posed the following famous conjecture.

Conjecture. Let $f$ be a non-constant entire function such that the hyper-order $\sigma_{2}(f)$ of $f$ is not a positive integer and $\sigma_{2}(f)<\infty$. If $f$ and $f^{\prime}$ share a finite value a $C M$, then

where $c$ is nonzero constant.

$$
\frac{f^{\prime}-a}{f-a}=c
$$

In fact, the conjecture for the case $a=0$ had been proved by R. Brück in [1]. From the differential equations

$$
\frac{f^{\prime}-a}{f-a}=e^{z^{n}}, \frac{f^{\prime}-a}{f-a}=e^{e^{z}}
$$

we see that when the hyper-order of $\sigma_{2}(f)$ is a positive integer or infinite, the conjecture does not hold.

The conjecture for the case that $f$ is of finite order had been proved by Gundersen and Yang [5] ; the case that $f$ is of infinite order with $\sigma_{2}(f)<\frac{1}{2}$ had been proved by Chen and Shon [3]. But the case $\sigma_{2}(f) \geq \frac{1}{2}$ is still open.

Under some additional assumptions, there are many results related to the Brück conjecture, see, e.g., [12, Theorem 1] and [16, Theorem 1.1].

In fact, to prove the conjecture is a hard work. Some have considered adding a deficient value condition to help solve the problem, see [15, Theorem 1]. Based on this, it is interesting to ask whether the Brück conjecture holds or not if the function $f$ is replaced by $n$-th powers $f^{n}$, since $f^{n}(n \geq 2)$ obviously satisfies the following deficient value condition,

$$
\Theta\left(0, f^{n}\right)=1-\varlimsup_{r \rightarrow \infty} \frac{\bar{N}\left(r, \frac{1}{f^{n}}\right)}{T\left(r, f^{n}\right)} \geq 1-\varlimsup_{r \rightarrow \infty} \frac{1}{n} \frac{N\left(r, \frac{1}{f^{n}}\right)}{T\left(r, f^{n}\right)} \geq 1-\frac{1}{n}=\frac{n-1}{n} .
$$

From (1.1), we see that the conjecture does not hold when $n=1$. Thus, we only need to discuss the problem when $n \geq 2$. Recently, Yang and Zhang [14] proved that the Brück conjecture holds for the function $f^{n}$, and the order restriction on $f$ is not needed if $n$ is relatively large. Actually, they proved the following result.

Theorem A. Let $f$ be a non-constant entire function, let $n \geq 7$ be an integer, and let $F=f^{n}$. If $F$ and $F^{\prime}$ share $1 C M$, then $F=F^{\prime}$, and $f$ assumes the form

$$
f(z)=c e^{\frac{1}{n} z}
$$

where $c$ is a non-zero constant.

It is natural to ask whether Theorem A still holds or not if the value 1 is replaced by a small function of $f$. In this work, we further study the problem and prove that the Brück conjecture holds for a special class of meromorphic functions. In fact, we obtain the following result. 
Theorem 1.1. Let $f$ be a transcendental meromorphic function with finitely many poles, let $n \geq 2$ be an integer, and let $\alpha=P e^{Q}\left(\neq \alpha^{\prime}\right)$ be an entire function such that the order of $\alpha$ is less than that of $f$, where $P, Q$ are two polynomials. If $f^{n}$ and $\left(f^{n}\right)^{\prime}$ share $\alpha C M$, then

$$
f=A e^{\frac{1}{n} z}
$$

where $A$ is a non-zero constant.

As a matter of fact, we deduce a more general result.

Theorem 1.2. Let $f$ be a transcendental meromorphic function with finitely many poles, and let $\alpha=P e^{Q}\left(\neq \alpha^{\prime}\right)$ be an entire function such that the order of $\alpha$ is less than that of $f$, where $P, Q$ are two polynomials. If $f$ has only multiple zeros and $f$ and $f^{\prime}$ share $\alpha C M$, then $f=f^{\prime}$ and $\alpha$ reduces to a polynomial.

Remark. If $Q$ is a constant, then Theorem 1.2 still holds even without the assumption that the order of $\alpha$ is less than that of $f$. Using a result of [9], this is easily deduced in a manner similar to our proof of Theorem 1.2.

\section{Some lemmas}

In order to prove our theorems, we need the following lemmas.

Lemma 2.1 ([8]). Let $\left\{f_{n}\right\}$ be a family of functions meromorphic (analytic) on the unit disc $\triangle$. If $a_{n} \rightarrow a,|a|<1$, and $f_{n}^{\sharp}\left(a_{n}\right) \rightarrow \infty$, then there exist

(a) a subsequence of $f_{n}$ (which we still write as $\left.\left\{f_{n}\right\}\right)$;

(b) points $z_{n} \rightarrow z_{0}\left|z_{0}\right|<1$;

(c) positive numbers $\rho_{n} \rightarrow 0$

such that $f_{n}\left(z_{n}+\rho_{n} \xi\right)=g_{n}(\xi) \rightarrow g(\xi)$ locally uniformly, where $g$ is a nonconstant meromorphic (entire) function on $\mathbb{C}$, such that

$$
\rho_{n} \leq \frac{M}{f_{n}^{\sharp}\left(a_{n}\right)},
$$

where $M$ is a constant which is independent of $n$.

Here, as usual, $g^{\sharp}(\xi)=\frac{\left|g^{\prime}(\xi)\right|}{1+|g(\xi)|^{2}}$ is the spherical derivative.

Lemma $2.2([7])$. Let $f$ be a meromorphic function of infinite order on $\mathbb{C}$. Then there exist points $z_{n} \rightarrow \infty$, such that for every $N>0, f^{\sharp}\left(z_{n}\right)>\left|z_{n}\right|^{N}$ if $n$ is sufficiently large.

Lemma $2.3([2])$. Let $f$ and $\alpha$ be meromorphic functions of finite order such that both of $f$ and $\alpha$ have finitely many poles, $f$ and $\alpha$ have no common poles and the order of $\alpha$ is less than the order of $f$. If $f$ and $f^{\prime}$ share $\alpha C M$, then $f^{\prime}-\alpha=c(f-\alpha)$ for some non-zero constant $c$. 


\section{Proof of Theorem 1.2}

We prove the main theorem with the method of J. Grahl and C. Meng [4, Theorem 1.1]. For the convenience of the reader, we present our proof in all detail.

We consider the function $F=\frac{f}{\alpha}$.

Case 1: $F$ has finite order.

Hence $f=F \alpha$ has finite order as well.

Set

$$
\mu=\frac{f^{\prime}-\alpha}{f-\alpha} .
$$

By Lemma 2.3, we obtain that $\mu$ is a constant. If $\mu=1$, we have $f=f^{\prime}$. Now, we suppose that $\mu \neq 1$. Rewriting (3.1) as

$$
f^{\prime}=\mu f+\alpha(1-\mu) .
$$

Now, we consider into two subcases.

Subcase 1.1. $f$ has finitely many zeros.

It follows from (3.2) that $f$ is an entire function. Since $f$ is of finite order, then we set $f=P_{1} e^{Q_{1}}$, where $P_{1}$ and $Q_{1}$ are two polynomials. Putting the form of $f$ into (3.2) yields

$$
\left[P_{1}^{\prime}+P_{1} Q^{\prime}-\mu P_{1}\right] e^{Q_{1}}=\alpha(1-\mu)=P e^{Q}(1-\mu) .
$$

It is obvious that $\operatorname{deg} Q_{1}=\operatorname{deg} Q$. It is obvious that $\rho(f)=\rho(\alpha)=\operatorname{deg} Q$, a contradiction.

Subcase 1.2. $f$ has infinitely many zeros.

Assume that $z_{0}$ is a zero of $f$. Noting that $f$ has only multiple zeros, we have $f^{\prime}\left(z_{0}\right)=0$. Substituting $z_{0}$ into $(3.2)$ yields

$$
0=\alpha\left(z_{0}\right)(1-\mu)=P\left(z_{0}\right) e^{Q\left(z_{0}\right)}(1-\mu),
$$

which implies that $P\left(z_{0}\right)=0$. Thus, the zeros of $f$ are also zeros of $P$. While $P$ is a polynomial and has only finitely many zeros, so $f$ has only finitely many zeros, which contradicts the assumption of Subcase 1.2.

Case 2. $F$ has infinite order.

By Lemma 2.2, there exist $w_{n} \rightarrow \infty$, such that for every $N>0$, if $n$ is sufficiently large

$$
F^{\sharp}\left(w_{n}\right)>\left|w_{n}\right|^{N} .
$$

Observing that $f$ has only finitely many poles and $\alpha$ has only finitely many zeros, then there exists a $r>0$ such that $f(z)$ is analytic and $\alpha(z) \neq 0$ in $|z| \geq r$. Let $D=\{z:|z| \geq r\}$, then $F(z)$ is analytic in $D$. In view of $w_{n} \rightarrow \infty$ as $n \rightarrow \infty$, without loss of generality we may assume $\left|w_{n}\right| \geq r+1$ for all $n$. Define $D_{1}=\{z:|z|<1\}$ and

$$
F_{n}(z)=F\left(w_{n}+z\right) .
$$


Then all $F_{n}(z)$ are analytic in $D_{1}$ and $F_{n}^{\sharp}(0)=F^{\sharp}\left(w_{n}\right) \rightarrow \infty$ as $n \rightarrow \infty$. It follows from Marty's criterion that $\left(F_{n}\right)_{n}$ is not normal at $z=0$.

Therefore, we can apply Lemma 2.1. Choosing an appropriate subsequence of $\left(F_{n}\right)_{n}$ if necessary, we may assume that there exist sequence $\left(z_{n}\right)_{n}$ and $\left(\rho_{n}\right)_{n}$ with $\left|z_{n}\right|<r<1$ and $\rho_{n} \rightarrow 0$ such that

$$
g_{n}(\zeta)=F_{n}\left(z_{n}+\rho_{n} \zeta\right)=\frac{f\left(w_{n}+z_{n}+\rho_{n} \zeta\right)}{\alpha\left(w_{n}+z_{n}+\rho_{n} \zeta\right)} \rightarrow g(\zeta)
$$

locally uniformly in $\mathbb{C}$ with a non-constant entire function $g$ whose zeros have multiplicity at least 2 and

$$
\rho_{n} \leq \frac{M}{F_{n}^{\sharp}(0)}=\frac{M}{F^{\sharp}\left(w_{n}\right)}
$$

for a positive number $M$. From (3.4) and (3.6), we deduce that, for every $N>0$, if $n$ is sufficiently large,

$$
\rho_{n} \leq M\left|w_{n}\right|^{-N}
$$

Differentiating (3.5) yields

$$
\begin{aligned}
g_{n}^{\prime}(\zeta) & =\rho_{n} \frac{f^{\prime}\left(w_{n}+z_{n}+\rho_{n} \zeta\right)}{\alpha\left(w_{n}+z_{n}+\rho_{n} \zeta\right)}-\rho_{n} \frac{f\left(w_{n}+z_{n}+\rho_{n} \zeta\right) \alpha^{\prime}\left(w_{n}+z_{n}+\rho_{n} \zeta\right)}{\alpha\left(w_{n}+z_{n}+\rho_{n} \zeta\right)^{2}} \\
& =\rho_{n} \frac{f^{\prime}\left(w_{n}+z_{n}+\rho_{n} \zeta\right)}{\alpha\left(w_{n}+z_{n}+\rho_{n} \zeta\right)}-\rho_{n} \frac{g_{n}(\zeta) \alpha^{\prime}\left(w_{n}+z_{n}+\rho_{n} \zeta\right)}{\alpha\left(w_{n}+z_{n}+\rho_{n} \zeta\right)} \rightarrow g^{\prime}(\zeta) .
\end{aligned}
$$

Noting (3.7), we deduce

$$
\rho_{n} \frac{g_{n}(\zeta) \alpha^{\prime}\left(w_{n}+z_{n}+\rho_{n} \zeta\right)}{\alpha\left(w_{n}+z_{n}+\rho_{n} \zeta\right)}=\rho_{n} \frac{g_{n}(\zeta)\left(P^{\prime}+P Q^{\prime}\right)\left(w_{n}+z_{n}+\rho_{n} \zeta\right)}{P\left(w_{n}+z_{n}+\rho_{n} \zeta\right)} \rightarrow 0
$$

Thus, we have

$$
\rho_{n} \frac{f^{\prime}\left(w_{n}+z_{n}+\rho_{n} \zeta\right)}{\alpha\left(w_{n}+z_{n}+\rho_{n} \zeta\right)} \rightarrow g^{\prime}(\zeta)
$$

Next, we prove that $g(\zeta)=1 \Rightarrow g^{\prime}(\zeta)=0$.

Suppose that $g\left(\zeta_{0}\right)=1$, then by Hurwitz's theorem, there exists a sequence $\left\{\zeta_{n}\right\}, \zeta_{n} \rightarrow \zeta_{0}$, such that (for $n$ sufficiently large)

$$
g_{n}\left(\zeta_{n}\right)=\frac{f\left(w_{n}+z_{n}+\rho_{n} \zeta_{n}\right)}{\alpha\left(w_{n}+z_{n}+\rho_{n} \zeta_{n}\right)}=1
$$

Thus $f_{n}\left(w_{n}+z_{n}+\rho_{n} \zeta_{n}\right)=\alpha\left(w_{n}+z_{n}+\rho_{n} \zeta_{n}\right)$. The assumption $f(z)=\alpha(z) \Rightarrow$ $f^{\prime}(z)=\alpha(z)$ implies that $f_{n}^{\prime}\left(w_{n}+z_{n}+\rho_{n} \zeta_{n}\right)=\alpha\left(w_{n}+z_{n}+\rho_{n} \zeta_{n}\right)$.

Then, by (3.9) we derive that

$$
g^{\prime}\left(\zeta_{0}\right)=\lim _{n \rightarrow \infty} \rho_{n} \frac{f^{\prime}\left(w_{n}+z_{n}+\rho_{n} \zeta_{n}\right)}{\alpha\left(w_{n}+z_{n}+\rho_{n} \zeta_{n}\right)}=\lim _{n \rightarrow \infty} \rho_{n}=0,
$$

which indicates that $g(\zeta)=1 \Rightarrow g^{\prime}(\zeta)=0$.

In the following, we prove $g(\zeta) \neq 1$. 
Suppose $\xi_{0}$ is a zero of $g-1$ with multiplicity $m(\geq 2)$, then $g^{(m)}\left(\xi_{0}\right) \neq 0$. Thus there exists a positive number $\delta$, such that

$$
g(\zeta) \neq 1, g^{\prime}(\zeta) \neq 0, g^{(m)}(\zeta) \neq 0
$$

on $D_{\delta}^{o}=\left\{z: 0<\left|\zeta-\xi_{0}\right|<\delta\right\}$.

Noting that $g \neq 1$, by Rouché theorem there exist $\xi_{n, j}(j=1,2, \ldots, m)$ on $D_{\delta / 2}=\left\{\xi:\left|\zeta-\xi_{0}\right|<\delta / 2\right\}$ such that

$$
g_{n}\left(\xi_{n, j}\right)=\frac{f\left(w_{n}+z_{n}+\rho_{n} \xi_{n, j}\right)}{\alpha\left(w_{n}+z_{n}+\rho_{n} \xi_{n, j}\right)}=1(j=1, \ldots, m) .
$$

That is $f\left(w_{n}+z_{n}+\rho_{n} \xi_{n, j}\right)=\alpha\left(w_{n}+z_{n}+\rho_{n} \xi_{n, j}\right)(j=1, \ldots, m)$. The fact that $f(z)=\alpha(z) \Rightarrow f^{\prime}(z)=\alpha(z)$ yields $f^{\prime}\left(w_{n}+z_{n}+\rho_{n} \xi_{n, j}\right)=\alpha\left(w_{n}+z_{n}+\right.$ $\left.\rho_{n} \xi_{n, j}\right)$.

Let

$$
H(\zeta)=f\left(w_{n}+z_{n}+\rho_{n} \zeta\right)-\alpha\left(w_{n}+z_{n}+\rho_{n} \zeta\right) .
$$

Thus, $H\left(\xi_{n, j}\right)=0(j=1, \ldots, m)$. We have

$$
\begin{aligned}
H^{\prime}\left(\xi_{n, j}\right) & =\rho_{n}\left[f^{\prime}\left(w_{n}+z_{n}+\rho_{n} \xi_{n, j}\right)-\alpha^{\prime}\left(w_{n}+z_{n}+\rho_{n} \xi_{n, j}\right)\right] \\
& =\rho_{n}\left[\alpha\left(w_{n}+z_{n}+\rho_{n} \xi_{n, j}\right)-\alpha^{\prime}\left(w_{n}+z_{n}+\rho_{n} \xi_{n, j}\right)\right] \\
& =\left.\rho_{n} e^{Q\left(w_{n}+z_{n}+\rho_{n} \xi_{n, j}\right)}\left[P-P^{\prime}-P Q^{\prime}\right]\right|_{\zeta=w_{n}+z_{n}+\rho_{n} \xi_{n, j}} .
\end{aligned}
$$

Since $\alpha \neq \alpha^{\prime}$, so $P-P^{\prime}-P Q^{\prime} \neq 0$. Thus, for $n$ large enough, we have

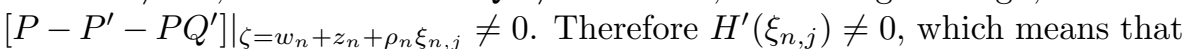
$\xi_{n, j}$ is a simple zero of $H(\zeta)(j=1, \ldots, m)$. That is $\xi_{n, j} \neq \xi_{n, i}(1 \leq i \neq j \leq m)$.

By (3.9), we have

$$
\begin{aligned}
K_{n}(\zeta) & =\rho_{n} \frac{f_{n}^{\prime}\left(w_{n}+z_{n}+\rho_{n} \zeta\right)-\alpha\left(w_{n}+z_{n}+\rho_{n} \zeta\right)}{\alpha\left(w_{n}+z_{n}+\rho_{n} \zeta\right)} \\
& =\rho_{n}\left[\frac{f^{\prime}\left(w_{n}+z_{n}+\rho_{n} \zeta\right)}{\alpha\left(w_{n}+z_{n}+\rho_{n} \zeta\right)}-1\right] \rightarrow g^{\prime}(\zeta),
\end{aligned}
$$

and $K_{n}\left(\xi_{n, j}\right)=0,(j=1, \ldots, m)$. From (3.10) we have

$$
\lim _{n \rightarrow \infty} \xi_{n, j}=\xi_{0}(j=1,2, \ldots, m) .
$$

Noting that $(3.11)$ and the fact that $K_{n}(\zeta)$ has $m$ zeros $\xi_{n, j}(j=1,2, \ldots, m)$ in $D_{\delta / 2}$, we obtain from the Hurwitz's theorem that $\xi_{0}$ is a zero of $g^{\prime}(\zeta)$ with multiplicity $m$, and thus $g^{(m)}\left(\xi_{0}\right)=0$. This is a contradiction. Hence $g(\zeta) \neq 1$.

With the Nevanlinna's second fundamental theorem, it is not difficult to deduce a contradiction. Thus, Case 2 cannot occur.

This completes the proof of Theorem 1.2.

Acknowledgment. The author is grateful to the referee for his(or her) valuable suggestions and comments. 


\section{References}

[1] R. Brück, On entire functions which share one value CM with their first derivatives, Results Math. 30 (1996), no. 1-2, 21-24.

[2] J. M. Chang and Y. Z. Zhu, Entire functions that share a small function with their derivatives, J. Math. Anal. Appl. 351 (2008), 491-496.

[3] Z. X. Chen and K. H. Shon, On conjecture of R. Brück concerning the entire function sharing one value CM with its derivative, Taiwanese J. Math. 8 (2004), no. 2, 235-244.

[4] J. Grahl and C. Meng, Entire functions sharing a polynomial with their derivatives and normal families, Analysis. (Munich) 28 (2008), no. 1, 51-61.

[5] G. G. Gundersen and L. Z. Yang, Entire functions that share one value with one or two of their derivatives, J. Math. Anal. Appl. 223 (1998), 88-95.

[6] W. Hayman, Meromorphic Functions, Clarendon Press, Oxford, 1964.

[7] X. J. Liu, S. Nevo, and X. C. Pang, On the kth derivative of meromorphic functions with zeros of multiplicity at least $k+1$, J. Math. Anal. Appl. 348 (2008), no. 1, 516-529.

[8] F. Lü, J. F. Xu, and A. Chen, Entire functions sharing polynomials with their first derivatives, Arch. Math. (Basel) 92 (2009), no. 6, 593-601.

[9] F. Lü and H. X. Yi, On the uniqueness problems of meromorphic functions and their linear differential polynomials, J. Math. Anal. Appl. 362 (2010), no. 2, 301-312.

[10] E. Mues and N. Steinmetz, Meromorphe Funktionen, die mit ihrer Ableitung Werte teilen, Manuscripta Math. 29 (1979), no. 2-4, 195-206.

[11] L. A. Rubel and C. C. Yang, Values shared by an entire function and its derivative, Complex analysis (Proc. Conf., Univ. Kentucky, Lexington, Ky., 1976), pp. 101-103. Lecture Notes in Math., Vol. 599, Springer, Berlin, 1977.

[12] J. Wang and H. X. Yi, Uniqueness theory of entire functions that share a small function with its differential polynomails, Indian J. Pure Appl. Math. 35 (2004), 1119-1129.

[13] C. C. Yang and H. X. Yi, The uniqueness theory of meromorphic functions, Mathematics and Its Applications, Science Press/Kluwer Acad. Publ, 2003.

[14] L. Z. Yang and J. L. Zhang, Non-existence of meromorphic solutions of a Fermat type functional equation, Aequationes Math. 76 (2008), no. 1-2, 140-150.

[15] J. L. Zhang and L. Z. Yang, Some results related to a conjecture of R. Brück concerning meromorphic functions sharing one small function with their derivatives, Ann. Acad. Sci. Fenn. Math. 32 (2007), no. 1, 141-149.

[16] _ A power of a meromorphic function sharing a small function with its derivative, Ann. Acad. Sci. Fenn. Math. 34 (2009), no. 1, 249-260.

Department of Mathematics

China University of Petroleum

Dongying, Shandong, 257061, P. R. China

E-mail address: 1vfeng18@gmail.com 